\title{
Modelamiento de propiedades magnéticas de aleaciones FeCox en películas delgadas
}

\author{
Modeling of magnetic properties of FeCox alloys in thin films \\ ${ }^{1,2}$ Elisabeth Restrepo-Parra, ${ }^{1}$ José Antonio Valencia Aricapa, ${ }^{2}$ Víctor Dahián Saldarriaga Montoya, \\ ${ }^{2}$ Sebastián Camilo Mendoza Rincón, ${ }^{2}$ Santiago Ospina Arroyave, ${ }^{2}$ Steven Vargas Villanueva, ${ }^{3} \mathrm{D}$. \\ M. Devia Narváez. \\ ${ }^{1}$ PCM Computational Applications, Universidad Nacional de Colombia Sede Manizales, Colombia \\ ${ }^{2}$ Laboratorio física del plasma, Universidad Nacional de Colombia Sede Manizales, Colombia \\ ${ }^{3}$ Universidad Tecnológica de Pereira, Pereira, Colombia \\ Correo-e: erestrepopalunal.edu.co
}

\begin{abstract}
Resumen-Se realizaron simulaciones de aleaciones de $\quad$ Fe$_{1}$ ${ }_{x} C_{x}$ en forma de películas delgadas empleando el método de Monte Carlo y el modelo de Heisenberg, con el fin de observar las variaciones de las propiedades magnéticas, la temperatura de transición y el campo coercitivo a medida que se varía la concentración de cobalto $(x)$. Para la construcción de las muestras se emplearon condiciones semi-periódicas. Se empleó además un Hamiltoniano que contiene términos correspondientes a la interacción entre primeros vecinos, y el término Zeeman. Dentro de la interacción entre primeros vecinos se consideraron tres tipos de constantes de intercambio: $\mathrm{Fe}-\mathrm{Fe}$, $\mathrm{Fe}-\mathrm{Co}$ y $\mathrm{Co}-\mathrm{Co}$. Además, se ubicaron átomos de cobalto aleatoriamente dentro de la red cristalina BCC de Fe. Se observó que tanto la temperatura crítica como el campo coercitivo tienden a disminuir a medida que se incrementa $x$.
\end{abstract}

Palabras clave - Campo coercitivo, Monte Carlo, propiedades magnéticas, temperatura crítica.

Abstract-Simulations of $\mathrm{Fe}_{1-\mathrm{x}} \mathrm{Co}_{\mathrm{x}}$ alloys as thin films using Monte Carlo Method and Heisenberg model have been carried out. Changes in magnetic properties, transition temperature, and coercive field as a function of the cobalt concentration $(x)$ were observed. Semi-periodic conditions were used for the samples construction. A Hamiltonian expression which contains terms corresponding to the nearest neighbors interaction and the Zeeman effect was used. Three types of exchange parameters were considered: $\mathrm{Fe}-\mathrm{Fe}$, $\mathrm{Fe}-\mathrm{Co}$ y $\mathrm{Co}-\mathrm{Co}$. Furthermore, cobalt atoms were randomly placed inside the $\mathrm{BCC}$ crystal lattice of $\mathrm{Fe}$. Decrease not only in the critical temperature but also in the coercive field was observes as the concentration $\boldsymbol{x}$ was increased.

Key Word - Coercive field, critical temperature, Magnetic properties, Monte Carlo.

\section{INTRODUCCIÓN}

Las aleaciones de FeCo han sido estudiadas gracias a que la presencia de cobalto en el hierro mejora parámetros físicos como la coercitividad magnética, la conductividad térmica y la conductividad eléctrica. Estos materiales son útiles en piezas polares de electroimanes, relés, equipos telefónicos y para cualquier aplicación en la que se desee una elevada intensidad magnética; además de ser uno de los materiales magnéticos con mejor desempeño [1]. En la literatura se encuentran reportes experimentales que se refieren a la producción de estos materiales por diferentes técnicas; $\mathrm{M}$. Pratzer y H.J. Elmers realizaron estudios de películas ultra delgadas de Fe-Co crecidas por la técnica de deposición de haces moleculares [2]. Entre sus resultados más importantes se encuentra la diferencia del comportamiento magnético de multicapas comparadas con el material en bloque. I. Jepu y sus colaboradores [3] realizaron estudios morfológicos de películas delgadas de Fe-Co crecidas por el método de arco de vacío termoiónico. Ellos realizaron el estudio de las propiedades magnéticas empleando el método no-destructivo de efecto Kerr magneto-óptico (MOKE).

Un factor importante que influye en las propiedades de aleaciones FeCo es la concentración de Co dentro del material debido a que la adición de éste en la estructura del hierro da lugar al incremento de la intensidad de saturación del hierro [1-3]. Aunque existe un buen número de trabajos experimentales [4], no se han realizado muchos estudios teóricos de modelamiento y simulación que permitan comprender la fenomenología que gobierna el comportamiento de estos materiales. Una de las técnicas más empleadas para hacer este tipo de simulaciones es el método de Monte Carlo. Este método ha sido ampliamente empleado para el estudio de propiedades magnéticas de diferentes materiales tales como grafeno y nano-partículas [5,6] entre otros. Esto se debe a que el método Monte Carlo es un 
algoritmo probabilístico que ayuda a reproducir la naturaleza aleatoria del comportamiento a nivel microscópico de los materiales. Por esta razón, las ventajas que presenta este algoritmo frente a otros modelos matemáticos exactos residen en que aquellas variables que determinan el comportamiento global del sistema son de tipo aleatorio (espines).

En este trabajo se presenta un estudio de las propiedades magnéticas en función de la temperatura y el campo magnético de aleaciones FeCo en forma de películas delgadas. Este estudio se realizó empleando el método de Monte Carlo combinado con el modelo de Heisenberg y el algoritmo de Metrópolis. Se obtuvieron propiedades tales como magnetización, susceptibilidad magnética y ciclos de histéresis, además del comportamiento crítico del material.

\section{METODOLOGÍA}

El Hierro y el Cobalto se caracterizan por tener espín neto de 2 y $3 / 2$ respectivamente. Para la construcción de este modelo se consideró una estructura BCC (del $\mathrm{Fe})$, incluyendo impurezas de Co de forma aleatoria, de acuerdo con el porcentaje deseado. En la figura 1 se presenta un esquema de la muestra construida a un $10 \%$ de Co. El Hamiltoniano empleado para realizar la simulación es [7-9]:

$$
H=-J_{i j} \sum_{i \neq j} \overrightarrow{S_{i}} \cdot \overrightarrow{S_{j}}-K_{a n} \sum_{i}\left(\overrightarrow{S_{i}} \cdot \hat{a}\right)^{2}-h \sum_{i} \overrightarrow{S_{i}} \cdot \hat{h}
$$

Donde $J_{i j}$ es la constante de intercambio entre primeros vecinos. Este parámetro puede tomar tres valores: para interacción $\mathrm{Fe}-\mathrm{Fe}$, para interacción Co-Co, para interacción Fe-Co. Es necesario tener en cuenta si la interacción es de tipo ferromagnético o si la interacción es de tipo antiferromagnética. Los mecanismos de intercambio para las fases FM y AFM son diferentes, y son vectores clásicos de espín de Heisenberg. $K_{a n}$ es la constante de anisotropía. â es el vector unitario en la dirección del eje fácil, $h$ y $\hat{h}$ son la magnitud y dirección del campo magnético externo aplicado respectivamente.

Se emplearon condiciones de frontera periódicas en el plano $x y$ y libres en el eje $z$ para simular el comportamiento de una película delgada [10]. Las simulaciones se llevaron a cabo en un proceso de enfriamiento desde una temperatura muy por encima de la temperatura de Curie del Fe (1043 K) [11] y del Co $(1388 \mathrm{~K})$ [12], hasta una temperatura de $100 \mathrm{~K}$, el cual inicia desde una configuración aleatoria de espines que corresponde a temperaturas altas. Los valores promedio se obtuvieron para $10^{4}$ pasos de Monte Carlo por espín, con el fin de llevar la muestra a condiciones de equilibrio. Las cantidades básicas termodinámicas obtenidas fueron la energía calculada a partir de la ecuación (1), la magnetización por sitio magnético ecuación (2), calor especifico $c$, ecuación (3) y susceptibilidad magnética $\chi$ ecuación (4). Además se obtuvieron ciclos de histéresis. Todas estas cantidades se calcularon para diferentes concentraciones de Cobalto [13].

$$
\begin{aligned}
& \frac{1}{L^{2} \times d} \sum_{i=1}^{L^{2} \times d} \overrightarrow{S_{i}} \\
& c=\left(1 / N k_{b} T^{2}\right)\left(\left\langle E^{2}\right\rangle-\langle E\rangle^{2}\right) \\
& \chi=\left(1 / N k_{b} T\right)\left(\left\langle m^{2}\right\rangle-\langle m\rangle^{2}\right)
\end{aligned}
$$

\section{RESULTADOS}

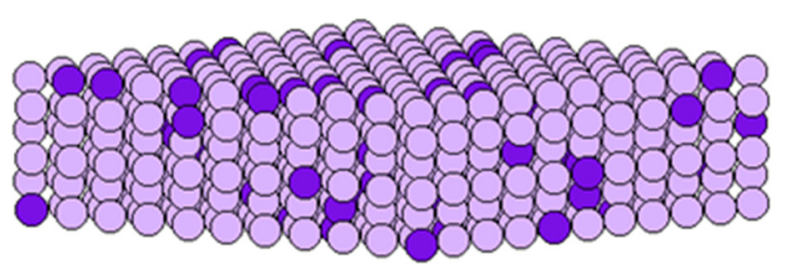

Figura 1. Película delgada de Fe con un porcentaje de Co del 10\%

En la figura 1 se observa un esquema de la muestra de $\mathrm{Fe}$ dopada con un $10 \%$ de Co. Se puede ver que los átomos de cobalto se organizan aleatoriamente, como lo requiere el modelo implementado. El estudio presentado en este trabajo está dedicado al análisis de las propiedades magnéticas de estos materiales.

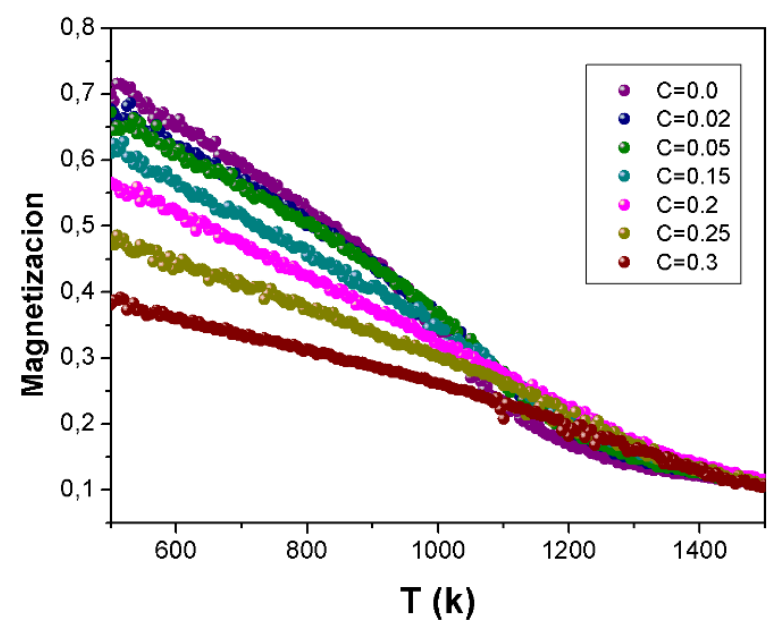

Figura 2. Magnetización en función de la temperatura para diferentes concentraciones de Cobalto

En la figura 2 se muestran curvas de magnetización en función de la temperatura para diferentes concentraciones de Co en la aleación $\mathrm{Fe}_{1-x} \mathrm{Co}_{x}$. Se observa que la magnetización disminuye a medida que se incrementa el porcentaje de Co. Esto se debe a que las interacciones entre los iones de Fe y Co son antiferromagnéticas, las cuales aumentan a expensas de las interacciones ferromagnéticas Fe-Fe. Este comportamiento se puede evidenciar en la figura 3 en la que se presenta la 
variación de la magnetización como función del porcentaje de Cobalto, a una temperatura de $600 \mathrm{~K}$. La disminución que se observa se debe a que al aumentar el porcentaje de Co, aumenta la cantidad de interacciones antiferromagnéticas.

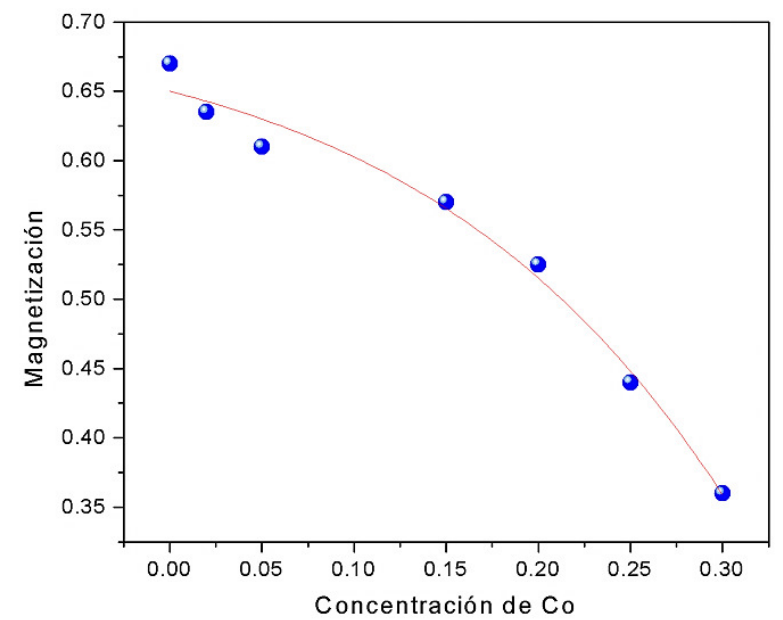

Figura 3. Magnetización en función de la concentración de Cobalto para $\mathrm{T}=600 \mathrm{~K}$.

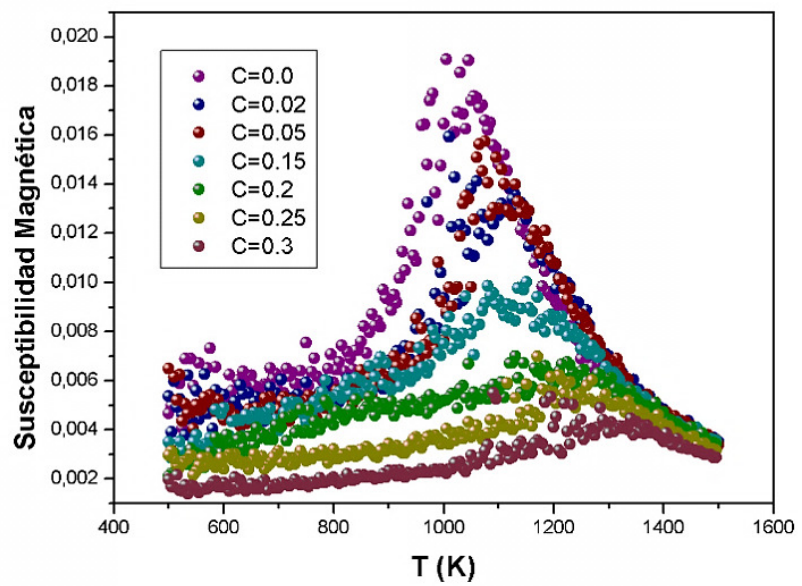

Figura 4. Susceptibilidad magnética en función de la temperatura

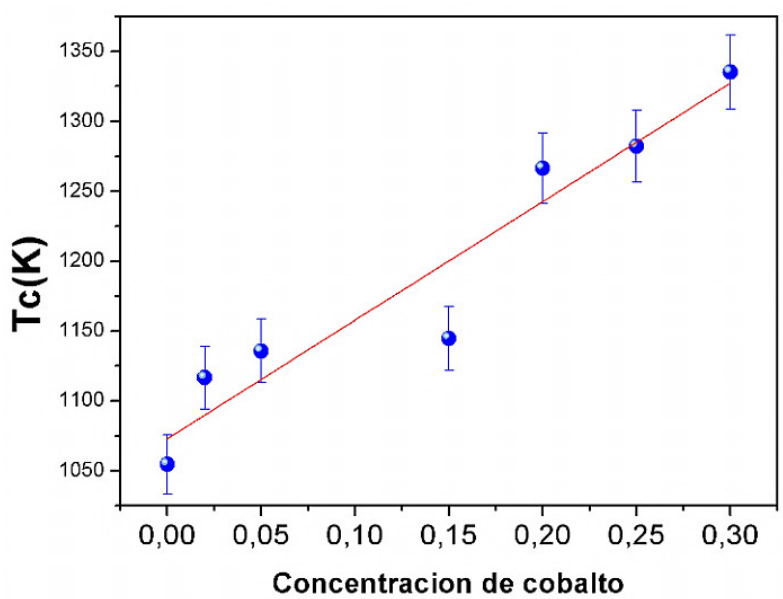

Figura 5. Tc en función del porcentaje de cobalto
La figura 4 presenta curvas de susceptibilidad magnética en función de la temperatura para diferentes concentraciones de Co. Se observa que la susceptibilidad disminuye a medida que se incrementa la concentración de Co; esto se debe al incremento de las interacciones anti-ferromagnéticas Fe-Co. También se puede evidenciar un corrimiento de la temperatura crítica hacia valores mayores como se muestra en la figura 5; Esto se debe que al incrementar la concentración de cobalto, las interacciones Co-Co también tienden a incrementar. Puesto que la temperatura de Curie del cobalto puro $\left(T_{c}=1388^{\circ} \mathrm{K}\right)$ es superior a la del hierro puro $\left(T_{c}=1043^{\circ} \mathrm{K}\right)$, la temperatura crítica de la aleación debe incrementar con el aumento de Co.

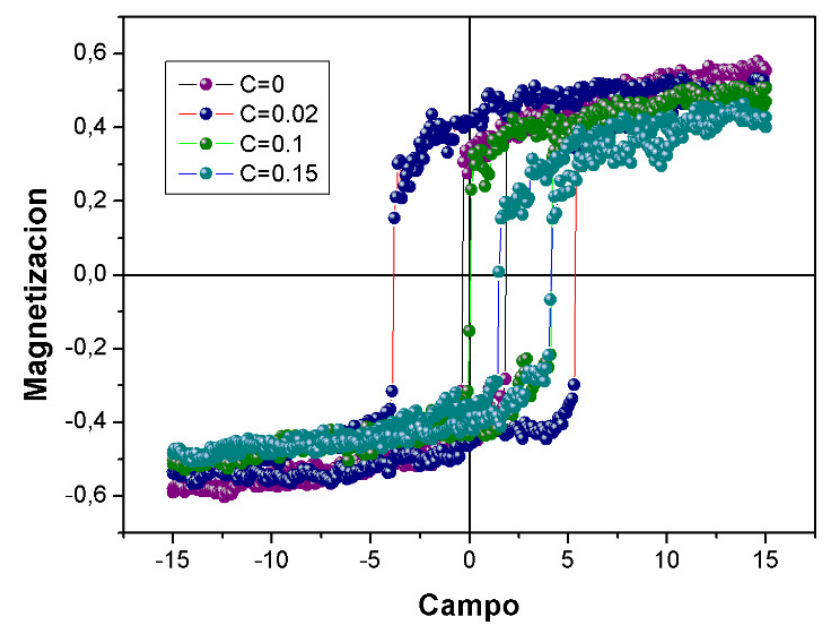

Figura 6. Ciclos de histéresis de la aleación $\mathrm{Fe}_{1-\mathrm{x}} \mathrm{Cox}_{\mathrm{x}}$ para diferentes concentraciones de $x$

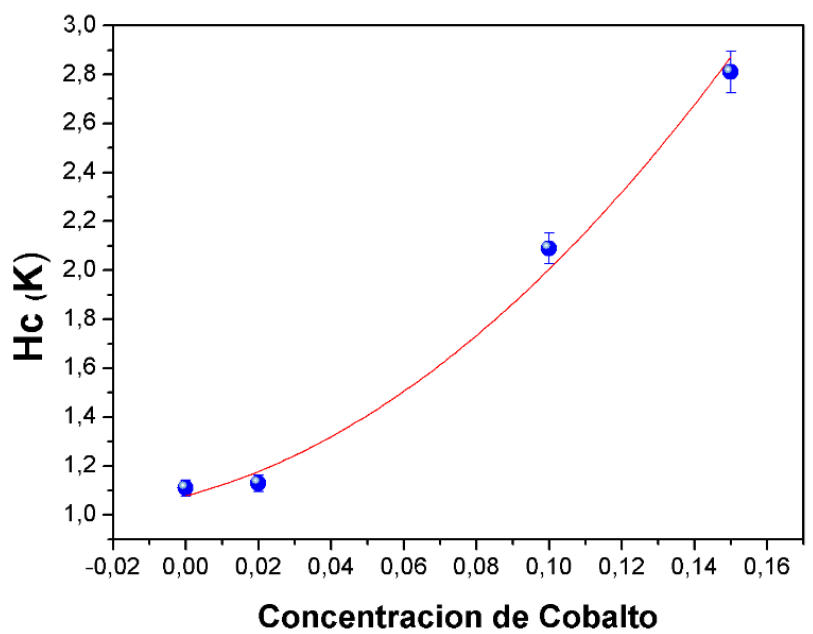

Figura $7 H_{c}$ en función del porcentaje de cobalto.

Por otro lado se estudió el comportamiento histerético del material. En la figura 6 se muestran ciclos de histéresis de la aleación $\mathrm{Fe}_{1-\mathrm{x}} \mathrm{Co}_{\mathrm{x}}$ para diferentes valores de $x$. Las curvas presentan un comportamiento ferromagnético. Se ve claramente la influencia del porcentaje de cobalto en el campo coercitivo en la figura 7 la cual presenta un incremento de $H_{c}$ 
con respecto a la concentración de Co. Esto quiere decir que al incrementar el porcentaje de $\mathrm{Co}$ el material se vuelve magnéticamente más duro. Nuevamente tiene que ver con que el cobalto puro es un material magnéticamente más duro que el hierro y tiene una temperatura de transición mayor. Al aumentar el cobalto, la aleación tiende a acercarse más a su comportamiento.

\section{CONCLUSIONES}

Se implementó un modelo empleando el método de Monte Carlo y el algoritmo Metrópolis combinado con el modelo de Heisenberg, para estudiar el comportamiento magnético de aleaciones $\mathrm{Fe}-\mathrm{Co}$ variando la concentración de cobalto. Se observó una fuerte influencia de este parámetro en la temperatura crítica y el campo coercitivo de la aleación.

\section{AGRADECIMIENTOS}

Los autores queremos agradecer a la Facultad de Ciencias Exactas y Naturales y a la dirección de investigaciones de la Universidad Nacional de Colombia - sede Manizales, por el apoyo económico bajo el proyecto 34705 - Estudio de las propiedades magnéticas de nanoestructuras y nanopartículas por medio del método Monte Carlo.

\section{REFERENCIAS}

[1] Caamaño-De Ávila1 Z. I., Consuegra Peña M.-A., Baró M. D., Suriñach S., "synthesis and characterization of nanostructured materials based on fe50co50 and Fe75Co25", CT\&F - Ciencia, Tecnología y Futuro, vol. 6, No. 2, pp. 33-44, dic. 2015.

[2] Pratzer, M., Elmers, H. J, "Magnetic and estructural investigation ultra-thin Co-Fe alloy films on W(110)", journal of magnetics and magnetic materials, vol. 272-276, pp. 1201-120,2004

[3] Jepu I., Porosnicu C., Lungu C.P., Mustata I., Luculescu C., Kuncser V., Iacobescu G., Marin A., Ciupina V., "Combinatorial FeCo thin film magnetic structures obtained by thermionic vacuum arc method", Surface \& Coatings Technology, vol. 240, pp. 344-352, 2014.

[4] Lin C.H., Chen W.H., Tsay J.S., Hong I.T., Chiu C.H., Huang H.S., "Structures and magnetic properties of $\mathrm{Co}$ and $\mathrm{CoFe}$ films prepared by magnetron sputtering" Thin Solid Films, vol. 519, pp. 8379-8383, 2011.

[5] Wang W., Ruijia Liu, Dan L., Luo X., "Monte Carlo simulation of magnetic properties of a nanographene bilayer in a longitudinal magnetic field", Superlattices and Microstructures, vol. 98, pp. 458472, 2016

[6] Jiang L., Zhang J., Chen Z., Feng Q., Huang Z., "Monte Carlo study of magnetic properties for the mixed spin-3/2 and spin-1 ferrimagnetic nanoparticles", Physica B, vol. 405, pp. 420-424, 2010 .
[7] Restrepo-Parra E., Bedoya-Hincapié C.M., Jurado F.J., Riano-Rojas J.C., Restrepo J., "Monte Carlo study of the critical behavior and magnetic properties of $\mathrm{La}_{2 / 3} \mathrm{Ca}_{1 / 3} \mathrm{MnO}_{3}$ thin films", Journal of Magnetism and Magnetic Materials, vol. 322, No. 21, pp. 3514-3518, 2010.

[8] Agudelo-Giraldo J.D., Restrepo-Parra E., Restrepo J., "Monte Carlo simulation of roughness effect on magnetic and magnetotransport behavior of $\mathrm{La}_{2 / 3} \mathrm{Ca}_{1 / 3} \mathrm{MnO}_{3} /$ $\mathrm{La}_{1 / 3} \mathrm{Ca}_{2 / 3} \mathrm{MnO}_{3}$ bilayers", Physica B: Condensed Matter, vol. 434, No. 1, pp. 149-154, 2014.

[9] Agudelo-Giraldo J.D., Restrepo-Parra E., Restrepo J., "Correlation between vacancies and magnetoresistance changes in FM manganites using the Monte Carlo method", Journal of Magnetism and Magnetic Materials, vol. 391, No. 1, pp. 129-135, 2015.

[10] Bedoya-Hincapié C.M., Restrepo-Parra E., Riaño Rojas J.C., Olaya-Flores J.J., Alfonso J.E., "Monte Carlo simulation of ferroelectric properties in bismuth titanate $\left(\mathrm{Bi}_{4} \mathrm{Ti}_{3} \mathrm{O}_{12}\right)$ thin films", Computational Materials Science, vol. 62, pp. 131$135,2012$.

[11] Yin J., Eisenbach M., Nicholson D.M., Rusanu A., "Effect of lattice vibrations on magnetic phase transition in bcc iron", Phys. Rev. B, vol. 86, 214423, 2012

[12] Singal C. M., Ciobanu G., Das T. P., "Ferromagnetic instabilities in cobalt: Critical temperature", Phys. Rev. B vol. 12, 2808, 1975.

[13] Restrepo-Parra E., Ramos-Rivera L., Londoño-Navarro J., "Magnetocaloric properties in $\mathrm{La}_{1-\mathrm{x}} \mathrm{Ca}_{\mathrm{x}} \mathrm{MnO}_{3}$ thin films: Monte Carlo simulations", Journal of Magnetism and Magnetic Materials, vol. 351, pp. 65-69, 2014. 